\title{
EFFECT OF THE CASCADE NEUTRALIZATION ENERGY ON THE SURFACE MODIFICATION BY THE IMPACT OF SLOW HIGHLY CHARGED IONS
}

\author{
MILENA MAJKIC ${ }^{1, \star}$, NATAŠA NEDELJKOVIĆ ${ }^{2}$, MARKO MIRKOVIĆ ${ }^{3}$ \\ ${ }^{1}$ Faculty of Technical Sciences, University of Priština, Kosovska Mitrovica, Serbia \\ ${ }^{2}$ Faculty of Physics, University of Belgrade, Belgrade, Serbia \\ ${ }^{3}$ University College of Civil Engineering and Geodesy, Belgrade, Serbia
}

\begin{abstract}
We consider the neutralization dynamics of slow highly charged ions in the interaction with a metal surface covered with a thin dielectric film from the standpoint of the surface modification. The quasi-resonant two-state vector model and the micro staircase model are used for the calculation of cascade neutralization energy for the $\mathrm{Ar}^{Z+}$, $\mathrm{Kr}^{Z+}$ and $\mathrm{Xe}^{Z+}$ ions. The population of several ionic Rydberg states at each step of the neutralization cascade is demonstrated. The correlation between the neutralization and the potential energy is presented for moderate and very low ionic velocities and the relevance of this relation for the surface nanostructure formation is briefly discussed for a different angle of incidence and different dielectric materials.
\end{abstract}

Keywords: Metal - dielectric surface, Highly charged ions, TVM, Rydberg states, Collision geometry, Neutralization energy, Surface nanostructures.

\section{INTRODUCTION}

Detailed study of the interaction of slow highly charged ions (HCI) with solid surfaces is of great interest for the analysis of the surface modification by their individual impact (Nedeljković et al., 2016). Depending on the ionic velocity and on the type of the solid surface, different surface structures (features) can be created (Aumayr et al., 2011; El-Said et al., 2008, 2016). During the ion-surface interaction, the electrons from the surface are captured into the intermediate ionic Rydberg states, which results in the neutralization cascade. The cascade neutralization energy has an important role in the surface modification, i.e., in the formation of different types of the surface nanostructures (Majkić et al., 2017). That is, the size of the created features is in a direct correlation with the ionic neutralization energy. For very low ionic velocity the size of the formed surface nanostructures is directly proportional to the ionic potential energy; for example, the pit volume is proportional to the potential energy of the $\mathrm{Xe}^{\mathrm{Z}+}$ ions interacting with $\mathrm{KBr}$ (Heller et al., 2008). However, for moderate ionic velocities, the surface modification is governed by the ionic neutralization energy which is less than the potential energy due to the incomplete neutralization. In that case, both the neutralization and the deposited kinetic energy contribute to the surface modification (Majkić et al., 2017, 2019).

The aim of the present article is twofold. The first aim is to correlate the neutralization and the potential energy of the HCI impinging upon a metal surface covered with a thin dielectric film for different collision geometry and film properties. The neutralization process will be considered within the quasi-resonant two-state vector model (TVM) for the population probabilities of the ionic intermediate Rydberg states and the micro staircase model for the multielectron neutralization. From the final ionic charge estimated quantum-mechanically within the proposed models, we directly obtain the required neutralization energy, the quantity necessary for the nanostructure formation. For the sake of simplicity, first we calculate the neutralization energy in the absence of dielectric film (metal-vacuum MV-system). The cascade neutralization energy, in the presence of dielectric film (metal-dielectric-vacuum, MDV-system), we calculate by using the principle of equivalence (Majkić et al., 2017), which correlates the quantities in the MVand the MDV-system via the concept of the effective core charge. The second aim is an analysis of the role of the cascade neutralization energy in the surface modification. That is, we correlate the size of the formed surface nanostructures and the obtained neutralization energy. The correlation of that type has been obtained between the size and the potential energy for the very low ionic velocities (Aumayr et al., 2011; El-Said et al., 2010, 2016).

This article is organized as follows. First, we present the TVM for the calculation of the population probabilities for the $\mathrm{Ar}^{Z+}, \mathrm{Kr}^{Z+}$ and $\mathrm{Xe}^{Z+}$ ions. Applying the micro staircase model we calculate the neutralization energy of these ions within the MDVsystem. In the subsequent analysis, we consider the correlation between the neutralization and the potential energy for $\mathrm{Xe}^{Z+}$, for different angle of incidence and different material of the dielectric film. We analyze the obtained correlation for $\mathrm{Ar}^{Z+}, \mathrm{Kr}^{Z+}$ and $\mathrm{Xe}^{\mathrm{Z+}}$ ions for two characteristic ionic velocities and discuss the corresponding relation between the surface nanostructure size and the

\footnotetext{
^ Corresponding author: milena.majkic@pr.ac.rs 
obtained neutralization energy. Atomic units $\left(e^{2}=\hbar=m_{e}=1\right)$ will be used throughout the paper unless indicated otherwise.

\section{NEUTRALIZATION DYNAMICS OF THE HCI IN THE MDV-SYSTEM}

We consider the HCI with initial charge $Z \gg 1$ approaching a metal surface covered with a thin dielectric film at velocity $v$. Two velocity regions will be considered: the moderate velocity case ( $v=0.25$ a.u.), characteristic for the crater formation within the MDV-system (Lake et al., 2011), and the very low velocity case $(v=0.1$ a.u.) characteristic for the hillocks created in the interaction of HCI with HOPG-surface (Aumayr et al., 2011). For the metal surface parameters, within the Sommerfeld model, we use the value of the work function $\phi=5 \mathrm{eV}$ and for the potential depth $U_{0}=15 \mathrm{eV}$. The dielectric film is characterized by the width $s_{0}$, and by the dielectric constants $\epsilon=2,4$ and 8 . We analyze the arbitrary collision geometry for the angles of incidence $\Phi_{i n}=\pi / 2,3 \pi / 8$ and $\pi / 4$.Due to the interaction of the HCI with solid surface the ionic charge decreases in time according to the cascade neutralization scenario $Q=Z \rightarrow Z-1 \rightarrow \ldots . \rightarrow Q_{\text {fin }}$, see Fig. (1).

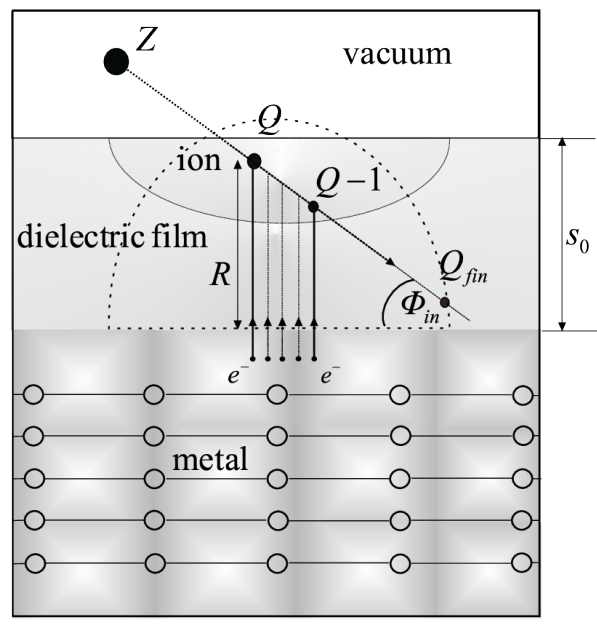

Figure 1. Cascade neutralization of the HCI in the MDV-system and the formation of the nanocrater; the hillock formation is presented by dashed line, schematically.

The neutralization process in the MDV-system we treat within the framework of the TVM and micro-staircase model (Majkić et al., 2017, 2019). According to the TVM the state of the active electron $e^{-}$is described by two state vectors $\left|\Psi_{1}(t)\right\rangle$ and $\left|\Psi_{2}(t)\right\rangle$, which simultaneously evolve in two opposite directions of time. The first state evolves from the initial state $\left|\Psi_{1}\left(t_{i n}\right)\right\rangle$ (electron in metal) towards the future, and the second state evolves towards the fixed final state $\left|\Psi_{2}\left(t_{\text {fin }}\right)\right\rangle$ (electron captured by the ion). The states of the active electron at the time $t$ (at ion-surface distance $R$ ) between the initial time $t_{\text {in }}$ and the final time $t_{\text {fin }}$ are expressed via intermediate eigenstates $\left|\mu_{M}(R)\right\rangle$ and $\left|v_{A}(R)\right\rangle$ of the inHamiltonian $\hat{H}_{1}(R)$ and the out-Hamiltonian $\hat{H}_{2}(R)$, respectively (Nedeljković et al., 2012; Nedeljković et al., 2016). By the ap- propriate expressions of the Hamiltonians $\hat{H}_{1}(R)$ and $\hat{H}_{2}(R)$ we take into account the polarization of the metal surface and the polarization of the ionic core, respectively, as well as the effect of the dielectric film (Majkić et al., 2017). The corresponding eigenenergies are $E_{M}=-\gamma_{M}^{2} / 2\left(\gamma_{M}\right.$ is the continous energy parameter) and $E_{A}=-\gamma_{A}^{2}(R) / 2=-\tilde{\gamma}_{A}^{2} / 2+(2 Z-1) / 4 R$, where $-\tilde{\gamma}_{A}^{2} / 2$ is the eigenenergy of the atomic Hamiltonian. The effect of the arbitrary collision geometry is taken into account by the Galilean invariance, i.e. by the translation factor in the function $\left|\Psi_{2}(t)\right\rangle$ (Nedeljković et al., 2012; Nedeljković et al., 2016) expressed via perpendicular velocity component $v_{\perp}$, and the energy shift $E_{M} \rightarrow E_{M}{ }^{\prime}=-\gamma_{M}^{\prime 2} / 2$ in the function $\left|\Psi_{1}(t)\right\rangle$, which depends on the parallel velocity component $v_{\|}$.

The population probability of the ionic intermediate Rydberg state within the MDV-system is governed by the corresponding quantity in the MV-system. In that case we use the relation between the transition probability density $T_{\mu_{M}^{\prime}, v_{A}}(t)$ and the mixed flux $I_{\mu_{M}^{\prime}, v_{A}}(t)$, the main quantity within the TVM:

$$
T_{\mu_{M}^{\prime}, v_{A}}(t)=\left|\int_{t_{i n}}^{t} I_{\mu_{M}, v_{A}}(t) d t\right|^{2} .
$$

The mixed flux we calculate through the fictive Firsov plane $S_{F}$ (Demkov \& Ostrovskii, 1975; Nedeljković \& Majkić, 2007), which separates the metal surface and the ionic region. The $S_{F}$ plane is positioned sufficiently far both from the surface and on the ionic core so that we use the asymptotic expressions of the wavefunctions $\Psi_{1}(\mathbf{r}, t)$ and $\Psi_{2}(\mathbf{r}, t)$ for the calculation of the mixed flux (Nedeljković et al., 2012). For an arbitrary collision geometry and moderate ionic velocities the electrons are captured quasi-resonantly from the overall metallic states $\left|\mu_{M}\right\rangle$ into the ionic Rydberg state $\left|v_{A}\right\rangle$ through the potential barrier formed between the surface and the ionic core. For very low ionic velocities the population process is resonant. In the more general velocity case, the intermediate population probability is given by $P_{v_{\mathrm{A}}}(R)=1-\exp \left[-T_{v_{\mathrm{A}}}(t)\right]$ (Nedeljković et al., 2016), where $T_{v_{\mathrm{A}}}(t)$ is calculated by the integration of the transition probability density $T_{\mu_{M}^{\prime}, v_{A}}(t)$ (Nedeljković et al., 2012):

$$
\begin{aligned}
T_{v_{A}}(t) & =2 \pi \mathcal{E}\left(R ; v_{\|}, v_{\perp}\right) T_{v_{A}}^{(0)} \frac{v_{\perp}}{\gamma_{A}|\tilde{\beta}|}\langle f\rangle_{\Omega_{\mathrm{k}^{\prime}}} \\
& \times f_{\gamma}\left(\gamma_{M}^{\prime}\right)\left[\gamma_{M}^{\prime}+\gamma_{A}(R)\right]^{2}\left(1+\frac{2 \tilde{\alpha}}{\tilde{\beta}} \frac{1}{R}\right) R^{2 \tilde{\alpha}} e^{-2 \tilde{\beta} R} .
\end{aligned}
$$

All quantities in (2) are defined for the quasi-resonant electron transitions, i.e. at ion-surface distance $R$ we have $\gamma_{M}^{\prime}=\gamma_{A}(R)$; the influence of the energy levels with $\gamma_{M}^{\prime} \neq \gamma_{A}(R)$ is expressed via $\mathcal{E}$ (Nedeljković et al., 2016). The quantity $\langle f\rangle_{\Omega_{k^{\prime}}}$ represents the angle-averaged Fermi-Dirac distribution of the electron momenta in solid (Nedeljković et al., 2012). The parameters $\tilde{\alpha}$ and $\tilde{\beta}$ in (2) are defined by $\tilde{\alpha}=Z / \tilde{\gamma}_{\mathrm{A}}-1 / 2+1 / 4 \gamma_{\mathrm{M}}^{\prime}$ and $\tilde{\beta}=\gamma_{\mathrm{M}}^{\prime}+\left(\tilde{\gamma}_{\mathrm{A}}-\right.$ $\left.\gamma_{\mathrm{M}}^{\prime}\right) / 2$, respectively. The explicit expressions for the factors $T_{v_{\mathrm{A}}}^{(0)}$ and $f_{\gamma}\left(\gamma_{\mathrm{M}}^{\prime}\right)$ are given in (Nedeljković et al., 2012).

Within the framework of the micro-staircase model, characteristic for the moderate ionic velocity (Nedeljković et al., 2016), 
the neutralization of the ion of the initial charge $Z$ and intermediate core charge $Q$, occurs through the neutralization cascades (macrosteps), which consist of the appropriate micro-cascades. The Rydberg levels with principal quantum numbers $n_{A}=n_{Z}-j+1$ of the particular micro-cascade (core charge $Q$ ) are populated with probabilities $P^{(Q, j)} \equiv \mathcal{P}_{n_{A}}=2 \sum_{l_{A}, m_{A}} P_{v_{A}}^{\max }$. For the probability $P_{v_{\mathrm{A}}}^{\max }$ we take the maximal value of the population probability $P_{v_{\mathrm{A}}}(R)$ at the neutralization distance $R=R_{c}^{N} \equiv R_{c}^{Q, j}\left(j \in\left[j_{\min }^{(Q)}, j_{\max }^{(Q)}\right]\right)$. The neutralization cascade $Q \rightarrow Q-1$, see Fig. (1), can be expressed by the following scheme (Nedeljković et al., 2016):

$$
\begin{aligned}
Q \rightarrow \quad & Q-P^{\left(Q, j_{\min }^{(Q)}\right)} \rightarrow \\
& Q-\left[P^{\left(Q, j_{\min }^{(Q)}\right)}+P^{\left(Q, j_{\min }^{(Q)}+1\right)}\right] \ldots \rightarrow \\
& Q-\sum_{j=j_{\min }^{(Q)}}^{j_{\max }^{(Q)}} P^{(Q, j)} \approx Q-1 .
\end{aligned}
$$

For very low velocity case the fine structure of the population process vanishes. Each macro cascade consists of only one micro step (staircase model) (Nedeljković et al., 2012).

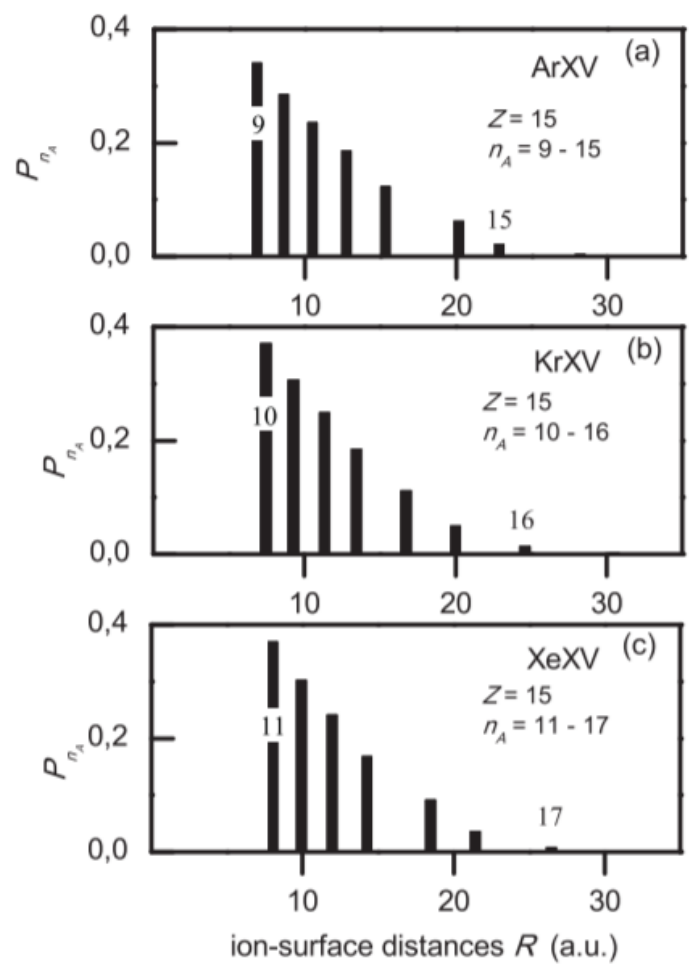

Figure 2. The first step of the cascade neutralization for $\mathrm{Ar}^{Z+}$, $\mathrm{Kr}^{Z+}$ and $\mathrm{Xe}^{\mathrm{Z}+}$ ions in the MV-system.

In Fig. (2) we present the first step $Q=Z \rightarrow Q=Z-1$ of the neutralization cascade within the MV-system of the ArXV, $\mathrm{KrXV}$ and XeXV ions $(Z=15)$ approaching a metal surface at velocity $v=0.25$ a.u. for $\Phi_{i n}=\pi / 2$. The considered step consists of the population of the series of Rydberg levels $n_{A}$ with probabilities $\mathcal{P}_{n_{A}}$ at neutralization distances $R=R_{c}^{N}$. In the considered case the Rydberg levels with principal quantum numbers from $n_{A}=15$ to $n_{A}=9$ for $\mathrm{ArXV}, n_{A}=16$ to $n_{A}=10$ for $\mathrm{KrXV}$ and $n_{A}=17$ to $n_{A}=11$ for XeXV are populated before other available levels, with $\sum \mathcal{P}_{n_{A}} \approx 1$. For the same ionic charge $Z=15$, different Rydberg levels of these ions are populated in each particular step, but at nearly the same ion-surface distances $R_{c}^{N}$ with the same population probability maxima, compare the neutralization distances for $n_{A}=$ 9, 10 and 11 in Fig. (2) (a), (b) and (c), respectively. In the next macro neutralization step $(Q=Z-1 \rightarrow Q=Z-2)$, not presented in figure, the population dynamics is similar to those presented in Fig. (2), but the number of populated levels decreases, because each successive macro step begins after the previous step ends, see figure 6 in (Nedeljković et al., 2016).

The neutralization dynamics within the MDV-system can be obtained from the corresponding one in the MV-system using the principle of equivalence based on the concept of the effective ionic charge $Z_{\text {eff }}$ (Majkić et al., 2017, 2019).

\section{RELATION BETWEEN THE CASCADE NEUTRALIZA- TION ENERGY AND THE POTENTIAL ENERGY AND THEIR ROLE IN THE SURFACE MODIFICATION}

The neutralization energy we compute by using the relation established for the MV-system: $W^{(Z, M V)}=W_{Z \text {,pot }}-W_{Q_{f i n}^{M V} \text {, pot }}$, where the first term $W_{Z \text {,pot }}$ represents the potential energy at the initial stages of the ionic trajectory, and the second term describes the potential energy at the last macro step of the neutralization cascade in front of the surface. The neutralization energy $W^{(Z, M D V)}$ in the MDV-system can be obtained from the corresponding quantities in MV-system according to the relation

$W^{(Z, M D V)}=W^{\left(Z_{e f f}, M V\right)}$,

where the effective ionic charge $Z_{\text {eff }}$ is calculated in (Majkic et al., 2017 ) for a given film properties $\left(\epsilon, s_{0}\right)$. We note that the final ionic charges for different geometries in the MV-system are the same due to the behaviour of the population probabilities maxima (see Fig. (2) and Fig.2 in (Majkić et al., 2019). The corresponding neutralization energies differ due to the difference in their potential energies.

The neutralization energy $W^{(Z, M D V)}$ obtained during the neutralization process $Z \rightarrow Q_{\text {fin }}$ is deposited into the surface. It represents the necessary amount of the energy for the nanofeatures formation. The surface modification simultaneously occurs with the proposed cascade neutralization of the ions. Consequently, the dielectric film is strongly perturbed and the corresponding dielectric constant $\epsilon$ is significantly lower in comparison to the unperturbed dielectric case.

The experimental results concerning the nanostructure formation by the impact of individual HCI are available for the very low ionic velocities and different types of surfaces and for the moderate velocities and the MDV-system. The experiments of the first class show the dependence of the size of the formed nanostructures on the HCI's potential energy. The impact of the HCI on different materials such as alkali and alkaline earth halides, polymers, oxides, HOPG, etc. has been studied (Aumayr et al., 
2011). For these experiments it is characteristic that the neutralization process is complete, i.e. $Q_{f i n}^{M V}=0$ and $W^{(Z, M V)}=W_{Z, p o t}$. For moderate ionic velocity, within the MDV-system as it has been obtained in experiments (Lake et al., 2011)), the neutralization is incomplete. For that reason, the corresponding neutralization energy differs from the ionic potential energy. It motivates us to correlate the neutralization and the potential energies of the ions.

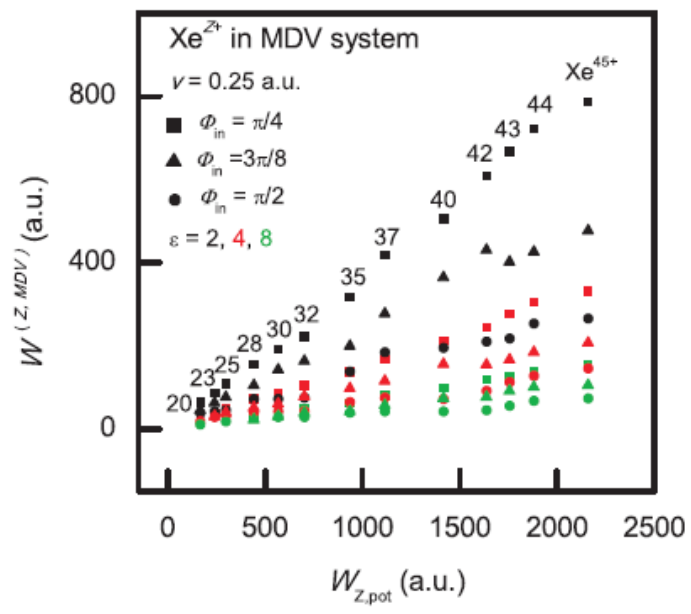

Figure 3. Neutralization energy $W^{(Z, M D V)}$ via initial potential energy $W_{Z \text {,pot }}$ of the $\mathrm{Xe}^{Z+}$ ions impinging at velocity $v=0.25$ a.u. upon a metal surface $\left(\phi=5 \mathrm{eV}, U_{0}=15 \mathrm{eV}\right)$ covered with a thin dielectric film $(\epsilon=2,4$ and 8$)$ of width $s_{0}=27$ a.u., for the angles of incidence $\Phi_{i n}=\pi / 2,3 \pi / 8$ and $\pi / 4$.

In Fig. (3) we present the cascade neutralization energy via initial potential energy for the $\mathrm{Xe}^{Z+}$ ions interacting with metal surface covered with a thin dielectric film for different collision geometries and different dielectric materials (dielectric constants $\epsilon=2,4$, and 8). We consider three angles of incidence $\Phi_{i n}=$ $\pi / 2,3 \pi / 8$ and $\pi / 4$. From Fig. (3) we can conclude that the neutralization energy depends on the combinations $\Phi_{\text {in }}$ and $\epsilon$. The same amount of the neutralization energy can be achieved with appropriate combination of these parameters; for example for $Z=40$ the same energy is for $\Phi_{i n}=3 \pi / 8$ and $\epsilon=8$ and $\Phi_{i n}=\pi / 2$ and $\epsilon=4$. The highest core charge $\mathrm{Xe}^{45+}$ has the highest neutralization energy for $\Phi_{i n}=\pi / 4$ and $\epsilon=2$, which corresponds to the highly perturbed dielectric. Having in mind that the formation of the particular surface nanostructure is determined by the available neutralization energy, we conclude that the nanostructure of the desired size can be obtained depending on the collision geometry and the surface type $(\epsilon)$.

In Fig. (4) we plot the neutralization energy via initial potential energy for the $\mathrm{Ar}^{Z+}, \mathrm{Kr}^{Z+}$ and $\mathrm{Xe}^{Z+}$ ions for two velocities $v=0.25$ a.u. and $v=0.1$ a.u. For the ionic velocity $v=0.25$ a.u. we consider the angle of incidence $\Phi_{i n}=\pi / 4$ and dielectric film of dielectric constants $\epsilon=2$. In the case $v=0.1$ a.u. we consider the neutralization energy in the absence of dielectric film $(\epsilon=1)$. The corresponding neutralization energy coincides with the ionic potential energy. From Fig. (4) we recog-

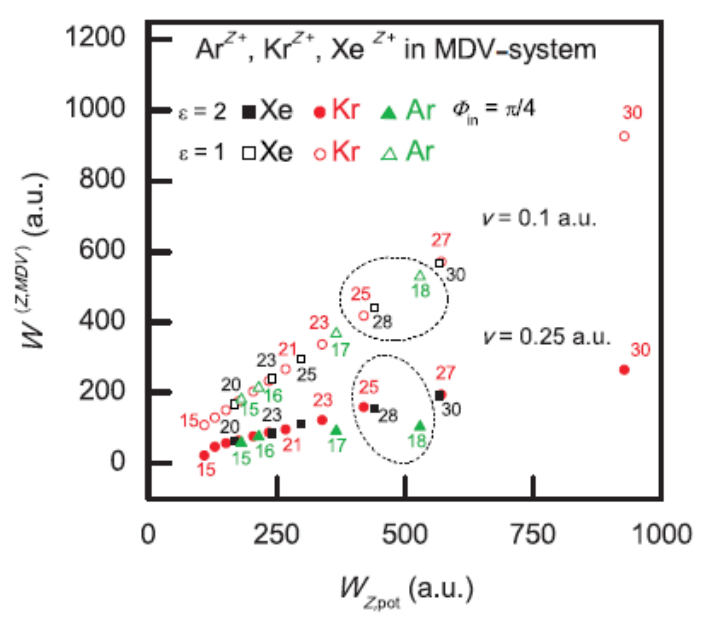

Figure 4. Neutralization energy $W^{(Z, M D V)}$ via initial potential energy $W_{Z \text {,pot }}$ of the $\mathrm{Ar}^{Z+}, \mathrm{Kr}^{Z+}$ and $\mathrm{Xe}^{Z+}$ ions impinging at velocity $v=0.25$ a.u. upon a metal surface $\left(\phi=5 \mathrm{eV}, U_{0}=15 \mathrm{eV}\right)$ covered with a thin dielectric film of width $s_{0}=27$ a.u. and $\epsilon=2$ for the angle of incidence $\Phi_{i n}=\pi / 4$, and for $v=0.1$ a.u. and $\epsilon=1$.

nize the effect of the ionic velocity; for example, for $v=0.25$ a.u. $W\left(\mathrm{Ar}^{18+}\right)<W\left(\mathrm{Kr}^{25+}\right) \approx W\left(\mathrm{Xe}^{28+}\right)$, while for $v=0.1$ a.u $W\left(\mathrm{Ar}^{18+}\right)>W\left(\mathrm{Kr}^{25+}\right) \approx W\left(\mathrm{Xe}^{28+}\right)$. This circumstance allows us to connect the neutralization energy to the size of the surface nanostructrues. For very slow ionic projectile when the potential energy plays a decisive role in the surface modification, the largest nanofeatures can be fabricated by the $\mathrm{Ar}^{18+}$ in comparison with the $\mathrm{Kr}^{25+}$ and $\mathrm{Xe}^{28+}$ ions. For slow, but moderate ionic velocity $(v=0.25$ a.u.), the neutralization energy together with the deposited kinetic energy influence to the nanostructure formation. In that case, the $\mathrm{Kr}^{25+}$ and the $\mathrm{Xe}^{28+}$ ions will create the nanostructures of larger size in comparison to the $\mathrm{Ar}^{18+}$ ions.

In Fig. 5 we present the dependence of the cascade neutralization energy on the potential energy for $\mathrm{Xe}^{Z+}$ ions impinging a metal surface covered with a thin dielectric film of width $s_{0}=27$ a.u. for two characteristic ionic velocities. We consider the very low ionic velocity $v=0.1$ a.u. case for the system parameters $\left(\Phi_{i n}, \epsilon\right)=(\pi / 2,1)$, Fig. $5(\mathrm{a})$, and the moderate ionic velocity $v=0.25$ a.u. case for the system parameters $\left(\Phi_{i n}, \epsilon\right)=(\pi / 2,2)$, Fig. 5 (b) (Majkić et al., 2019). In Figs. 5 (a) and (b), as an inset, we present the experimental results for the hillock diameter $r$ within the HOPG-surface ((Aumayr et al., 2011)), and the crater depth $d$ for the system $\mathrm{Co}+\mathrm{Al}_{2} \mathrm{O}_{3}$ ((Lake et al., 2011)), respectively, obtained for the two considered velocities. Nanostructure sizes are presented in atomic units: atomic unit length $=0.0529$ nm.

From Fig. 5 we see the similar linear dependence of the neutralization energy $W^{(Z, M D V)}$ on the potential energy $W_{Z, p o t}$ and the surface nanostucture sizes $r$ and $d$ expressed via $W_{Z, p o t}$. According to the observed functional dependencies we conclude that the neutralization energy crucially determines the size of the created surface features (craters and hillocks). We note that the contribu- 

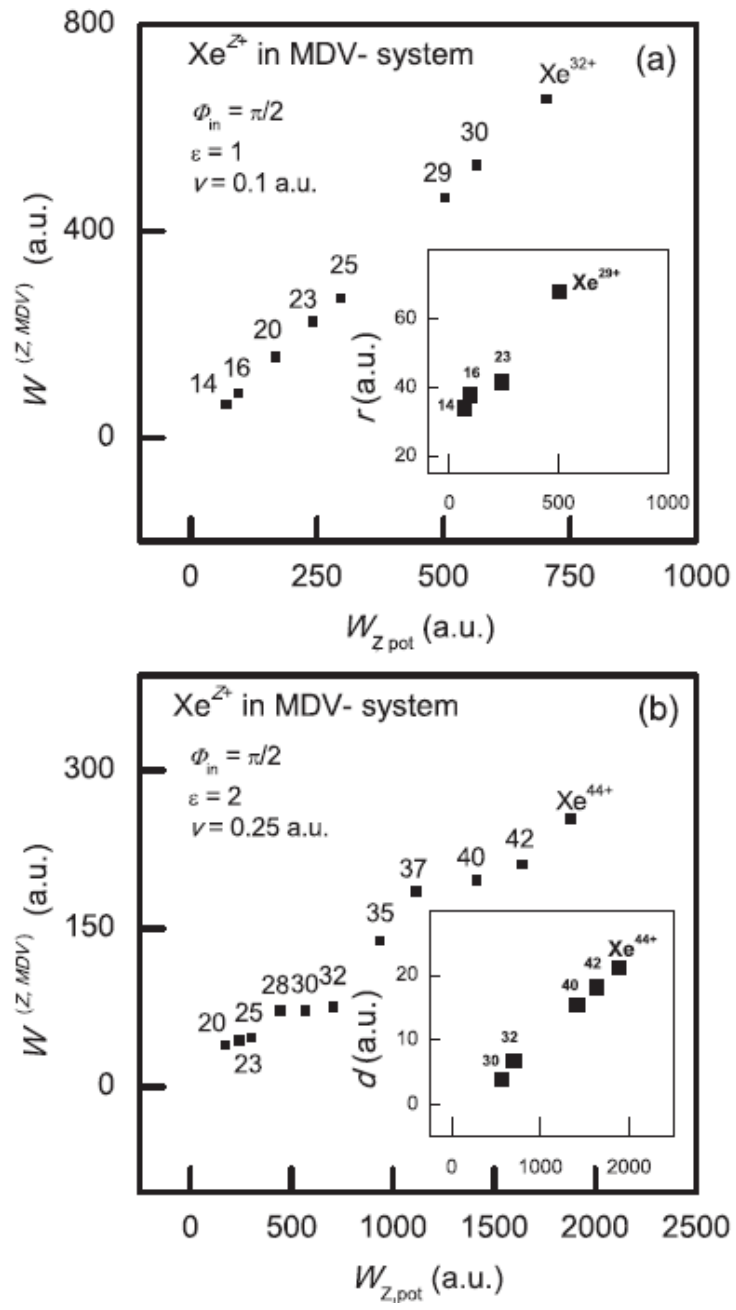

Figure 5. Neutralization energy $W^{(Z, M D V)}$ via potential energy $W_{Z, p o t}$ for $\mathrm{Xe}^{Z+}$ ion for $\Phi_{i n}=\pi / 2$. (a) Velocity $v=0.1$ a.u. and $\epsilon=1$; Inset: hillock diameter $r$ obtained experimentally (Aumayr et al., 2011) within HOPG system. (b) Velocity $v=0.25$ a.u. and $\epsilon=2$; Inset: crater depth $d$ obtained experimentally (Lake et al., 2011) within the $\mathrm{Co}+\mathrm{Al}_{2} \mathrm{O}_{3}$.

tion of the neutralization energy in the surface modification depends on the ionic velocity; for very low ionic velocities neutralization energy $W^{(Z, M D V)} \approx W_{Z, p o t}$ directly governed the surface features size, while for the moderate ionic velocities the quantity $W^{(Z, M D V)}<W_{Z, p o t}$ represents only the part of the deposited energy necessary for the considered nanostructure formation. The effect of the neutralization energy in the surface modification also depends on the surface type and the collision geometry.

\section{CONCLUDING REMARKS}

In this paper, for the first time, we presented the detailed analysis of the relation between the cascade neutralization energy $W^{(Z, M D V)}$ and the initial potential energy $W_{Z \text {,pot }}$ of the HCI (charge $Z$ ) impinging upon a metal surface covered with a thin dielectric film for arbitrary collision geometry and film properties. We consider the $\mathrm{Ar}^{Z+}, \mathrm{Kr}^{Z+}$ and $\mathrm{Xe}^{Z+}$ ions for dielectric constants $\epsilon=2,4$ and 8 , and for three different angles of incidence $\Phi_{\text {in }}=\pi / 2,3 \pi / 8$ and $\pi / 4$. By the impact of the individual HCI upon a surface the nanostuctures (craters and hillocks) are created. We discussed the role of the cascade neutralization energy for the surface modification.

From the similarity of the obtained linear dependence between the quantities $W^{(Z, M D V)}$ and $W_{Z, p o t}$ and the corresponding experimental relation between the size of the nanostructures and the potential energy, we conclude that the nanostructure sizes are directly governed by the cascade neutralization energy. Concerning the influence of the projectile velocity on the cascade neutralization energy, we demonstrated the velocity dependence of the effect of the neutralization energy on the surface modification. For very low ionic velocity characteristic for the complete neutralization, the potential energy has the main role in the creation of the surface nanofeatures. For low, but moderate ionic velocity, the cascade neutralization energy, which is less than the potential energy due to incomplete neutralization, plays a decisive role in the surface modification.

\section{ACKNOWLEDGMENTS}

This work was supported in part by the Ministry of Education, Science and Technological Development of the Republic of Serbia, Serbia, under the grants NO 171029, 171016.

\section{REFERENCES}

Aumayr, F., Facsko, S., El-Said, A. S., Trautmann, C., \& Schleberger, M. 2011. Single ion induced surface nanostructures: a comparison between slow highly charged and swift heavy ions. Journal of Physics: Condensed Matter, 23(39), p. 393001. doi:10.1088/0953-8984/23/39/393001.

Demkov, Y. N. \& Ostrovskii, V. N. 1975. Zh. Eksp. Teor. Fiz, 69, pp. 1582.

El-Said, A. S., Heller, R., Aumayr, F., \& Facsko, S. 2010. Pyramidal pits created by single highly charged ions in BaF 2 single crystals. Physical Review B, 82(3). doi:10.1103/physrevb.82.033403.

El-Said, A. S., Wilhelm, R. A., Heller, R., et al. 2008. Creation of Nanohillocks on CaF 2 Surfaces by Single Slow Highly Charged Ions. Physical Review Letters, 100(23). doi:10.1103/physrevlett.100.237601.

El-Said, A. S., Wilhelm, R. A., Heller, R., et al. 2016. Tuning the Fabrication of Nanostructures by Low-Energy Highly Charged Ions. Physical Review Letters, 117(12). doi:10.1103/physrevlett.117.126101.

Heller, R., Facsko, S., Wilhelm, R. A., \& Möller, W. 2008. Defect Mediated Desorption of the $\mathrm{KBr}(001)$ Surface Induced by Single Highly Charged Ion Impact. Physical Review Letters, 101(9). doi:10.1103/physrevlett.101.096102.

Lake, R., Pomeroy, J. M., Grube, H., \& Sosolik, C. E. 2011. Charge State Dependent Energy Deposition by Ion Impact. Physical Review Letters, 107(6). doi:10.1103/physrevlett.107.063202. 
Majkić, M. D., Nedeljković, N. N., \& Dojčilović, R. J. 2017. Interaction of slow highly charged ions with a metal surface covered with a thin dielectric film. The role of the neutralization energy in the nanostructures formation. Materials Research Express, 4(9), p. 95027. doi:10.1088/2053-1591/aa8bc7.

Majkić, M. D., Nedeljković, N. N., \& Mirković, M. A. 2019. Neutralization energy contribution to the nanostructure creation by the impact of highly charged ions at arbitrary angle of incidence upon a metal surface covered with a thin dielectric film. Vacuum, 165, pp. 62-67. doi:10.1016/j.vacuum.2019.04.002.

Nedeljković, N. N. \& Majkić, M. D. 2007. Intermediate stages of the Rydberg-level population of multiply charged ions escaping solid surfaces. Physical Review A, 76(4). doi:10.1103/physreva.76.042902.
Nedeljković, N. N., Majkić, M. D., Božanić, D. K., \& Dojčilović, R. J. 2016. Dynamics of the Rydberg state population of slow highly charged ions impinging a solid surface at arbitrary collision geometry. Journal of Physics B: Atomic, Molecular and Optical Physics, 49(12), p. 125201. doi:10.1088/09534075/49/12/125201.

Nedeljković, N. N., Majkić, M. D., \& Galijaš, S. M. D. 2012. Grazing incidence collisions of multiply charged ions on solid surfaces. Influence of the formation of intermediate Rydberg states. Journal of Physics B: Atomic, Molecular and Optical Physics, 45(21), p. 215202. doi:10.1088/09534075/45/21/215202. 